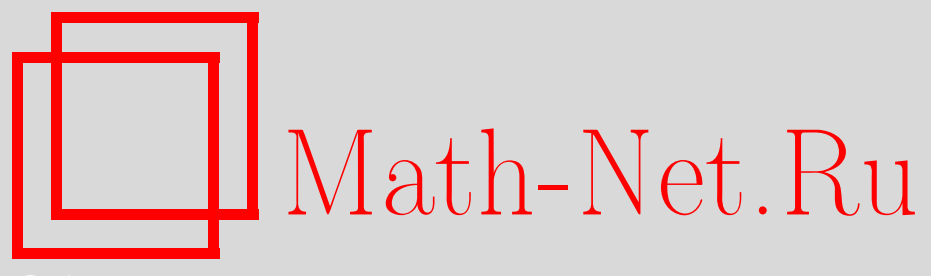

C. М. Натанзон, А. М. Пратусевич, Классификация mспинорных клейновых поверхностей, УМН, 2016, том 71, выпуск 2, 211-212

DOI: https://doi.org/10.4213/rm9695

Использование Общероссийского математического портала Math-Net.Ru подразумевает, что вы прочитали и согласны с пользовательским соглашением http://www . mathnet.ru/rus/agreement

Параметры загрузки:

IP : 54.210 .77 .194

26 апреля 2023 г., 14:45:14

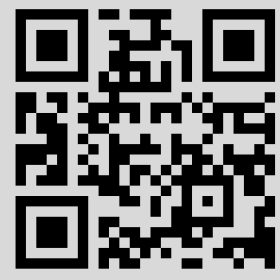




\section{Классификация $m$-спинорных клейновых поверхностей}

\section{С. М. Натанзон, А. М. Пратусевич}

Клейновы поверхности - это аналог римановых поверхностей для неориентируемых поверхностей и/или поверхностей с границей [1], [4]. Клейновы поверхности представляются в виде $P / \tau$, где $\tau: P \rightarrow P$ - антиголоморфная инволюция римановой поверхности $P$. Категория клейновых поверхностей изоморфна категории вещественных алгебраических кривых. Топологические инварианты связной клейновой поверхности $P / \tau$ исчерпываются ее (алгебраическим) родом $g=g(P)$, числом овалов, т. е. компонент связности границы: $k=k(P / \tau)=|\partial(P / \tau)|$, и ориентируемостью $\varepsilon=\varepsilon(P / \tau)$. Положим $\varepsilon=1$, если поверхность ориентируема (в этом случае $1 \leqslant k \leqslant g+1, k \equiv g+1 \bmod 2)$, и $\varepsilon=0$, если поверхность неориентируема (в этом случае $0 \leqslant k \leqslant g$ ). Наряду с этими инвариантами удобно рассматривать геометрический род $\widetilde{g}=\widetilde{g}(P / \tau)$. Если $\varepsilon=1$, то $\widetilde{g}-$ это число ручек, которые надо приклеить к сфере с дырками, чтобы она стала гомеоморфной $P / \tau$. Если $\varepsilon=0$, то $\widetilde{g}-$ это половина числа листов Мёбиуса, которые надо приклеить к сфере с дырками, чтобы она стала гомеоморфной $P / \tau$. Далее мы считаем, что $\widetilde{g}>1$ и, в частности, $g>1$.

$m$-спинорное расслоение на римановой или клейновой поверхности $S$ - это линейное расслоение $e: L \rightarrow S$ такое, что расслоение $e^{\otimes m}: L^{\otimes m} \rightarrow S$ изоморфно кокасательному расслоению. Пространство модулей $m$-спинорных расслоений на римановых поверхностях описано в [2], [7], [8]. Эти расслоения и их пространства модулей естественно возникают в математической физике [9]. Пространство модулей компактных римановых поверхностей $P$ рода $g>1$ с $m$-спинорным расслоением связно при нечетном $m$ и распадается на две компоненты связности при четном $m$. Эти компоненты определяются инвариантом Арфа $\delta=\delta(P, e) \in\{0,1\}$. На каждой римановой поверхности рода $g$ имеется $m^{2 g} m$-спинорных расслоений, среди которых (при четном $m$ ) $2^{-1-g} m^{2 g}\left(2^{g}+1\right)$ расслоений с $\delta=0$ и $2^{-1-g} m^{2 g}\left(2^{g}-1\right)$ расслоений с $\delta=1$. Наша цель - найти топологические инварианты $m$-спинорных расслоений на клейновых поверхностях геометрического рода $\widetilde{g}>1$, найти число таких расслоений и описать пространство модулей клейновых поверхностей с $m$-спинорным расслоением. Для $m=2$ это сделано в [3], [6].

Теорема 1. Всякая компонента связности пространства модулей клейновых поверхностей рода g с т-спинорным расслоением гомеоморфна $\mathbb{R}^{3 g-3} / \mathrm{Mod}$, где Mod дискретная группа.

Теорема 2. Если $m$ нечетно, то т-спинорное расслоение существует на клейновой поверхности $P / \tau$ тогда и толъко тогда, когда $g(P) \equiv 1 \bmod m ; n р и$ этом число таких расслоений равно $\mathrm{m}^{g}$. Более того, для нечетного $m$ пространство модулей всех клейновых поверхностей рода $g$ с т-спинорным расслоением связно.

Далее мы считаем, что $m$ четно.

Теорема 3. Если т четно, то т-спинорное расслоение существует на клейновой поверхности $P / \tau$ тогда и только тогда, когда $g(P) \equiv 1 \bmod (m / 2)$; число таких

Со стороны первого автора статья подготовлена в ходе проведения работ по проекту № 15-01-0052 в рамках Программы "Научный фонд Национального исследовательского университета "Высшая школа экономики" (НИУ ВШЭ)" в 2015-2016 гг. и с использованием средств субсидий на государственную поддержку ведущих университетов Российской Федерации в целях повышения их конкурентоспособности среди ведущих мировых научно-образовательных центров, выделенной НИУ ВШЭ. Исследование второго автора выполнено при поддержке Leverhulme Trust (грант RPG-057).

DOI: $10.4213 / \mathrm{rm} 9695$ 
расслоений с инвариантом Арфа б равно: 1) $m^{g} / 2$, если $\varepsilon=k=0$; 2) $2^{k-2} m^{g}$, если $\varepsilon=0, k>0$ или $m \equiv 0 \bmod 4, \varepsilon=1 ; 3)\left(2^{k-1}+1\right) m^{g} / 2$, если $m \equiv 2 \bmod 4, \varepsilon=1$, $\delta=0 ; 4)\left(2^{k-1}-1\right) m^{g} / 2$, если $m \equiv 2 \bmod 4, \varepsilon=1, \delta=1$.

Ограничение спинорного расслоения на овал может быть как тривиальным, так и нетривиальным расслоением. Обозначим через $k_{0}=k_{0}(P / \tau, e)$ и $k_{1}=k_{1}(P / \tau, e)$ число овалов с тривиальным и нетривиальным ограничением соответственно.

Теорема 4. Пространство модулей клейновых поверхностей с расслоением фиксированного типа $\left(g, \varepsilon, m, \delta, k_{0}, k_{1}\right)$ непусто тогда и только тогда, когда $k_{1} m / 2 \equiv$ $(1-g) \bmod m$. При $\varepsilon=0$ это пространство связно. На любой клейновой поверхности типа $\left(g, k_{0}+k_{1}, 0\right)$ существует $C_{k}^{k_{1}} m^{g} / 2$ расслоений типа $\left(g, 0, m, \delta, k_{0}, k_{1}\right)$.

Далее мы считаем, что $k_{1} m / 2 \equiv(1-g) \bmod m$ и $\varepsilon=1$. В этом случае существуют дополнительные топологические инварианты. Прежде всего, это инвариант Арфа $\widetilde{\delta}=\widetilde{\delta}(P / \tau)$ римановой поверхности $P / \tau \backslash \partial(P / \tau)$. Кроме того, отношение подобия делит овалы на два класса. Овалы $c_{1}$ и $c_{2}$ называются подобными [5], если существует пересекающий их простой замкнутый контур $d$ такой, что ограничение расслоения на него нетривиально и $\tau(d)=d$. Выберем один из классов подобия и обозначим через $k_{0}^{0}$ и $k_{1}^{0}$ число овалов этого класса, ограничение расслоения на которые тривиально и нетривиально соответственно. Положим $k_{i}^{1}=k_{i}-k_{i}^{0}$. (Набор инвариантов $\left(k_{0}^{0}, k_{1}^{0}, k_{0}^{1}, k_{1}^{1}\right)$ определен с точностью до замены $k_{i}^{j} \mapsto k_{i}^{1-j}$.)

Теорема 5. Пространство модулей расслоений с инвариантами $\left(g, \widetilde{\delta}, m, k_{0}^{0}, k_{1}^{0}\right.$, $\left.k_{0}^{1}, k_{1}^{1}\right)$ связно. Если $m \equiv 0 \bmod 4$ или $m \equiv 2 \bmod 4, k_{0}>0$, то число расслоений такого типа на произвольной клейновой поверхности отлично от нуля тогда и только тогда, когда $\widetilde{\delta}=0$, и равно в этом случае $2^{1-k} m^{g} C_{k}^{k_{1}} C_{k_{0}}^{k_{0}^{0}} C_{k_{1}}^{k_{1}^{0}}$. При $m \equiv 2$ $\bmod 4$ u $k_{0}=0$ число структур типа $\left(g, \widetilde{\delta}, m, 0, k_{1}^{0}, 0, k_{1}^{1}\right)$ на произвольной клейновой поверхности типа $\left(g, k_{1}^{0}+k_{1}^{1}, 1\right)$ равно $\left(2^{-k}+2^{-(g+k+1) / 2}\right) m^{g} C_{k}^{k_{1}} C_{k_{0}}^{k_{0}^{0}} C_{k_{1}}^{k_{1}^{0}}$, если $\widetilde{\delta}=0$, $u\left(2^{-k}-2^{-(g+k+1) / 2}\right) m^{g} C_{k}^{k_{1}} C_{k_{0}}^{k_{0}^{0}} C_{k_{1}}^{k_{1}^{0}}$, eсли $\widetilde{\delta}=1$.

\section{Список литературы}

[1] N.L. Alling, N. Greenleaf, Foundation of the theory of Klein surfaces, Lecture Notes in Math., 219, Springer-Verlag, Berlin-New York, 1971, ix+117 pp. [2] T. J. Jarvis, Internat. J. Math., 11:5 (2000), 637-663. [3] С. М. Натанзон, УМH, 44:3(267) (1989), 165-166. [4] С. М. Натанзон, УМH, 45:6(276) (1990), 47-90. [5] С. М. Натанзон, УMH, 54:6(330) (1999), 3-60. [6] С. М. Натанзон, Модули римановых поверхностей, вещественных алгебраических кривых и их супераналоги, МЦНМО, М., 2003, 176 с. [7] С. М. Натанзон, А. М. Пратусевич, УMH, 60:2(362) (2005), 169-170. [8] S. Natanzon, A. Pratoussevitch, J. Lie Theory, 19:1 (2009), 107-148. [9] E. Witten, Topological methods in modern mathematics (Stony Brook, NY, 1991), Publish or Perish, Houston, TX, 1993, 235-269.

\section{M. Натанзон (S. M. Natanzon)}

Национальный исследовательский университет

"Высшая школа экономики"; НИУ ВШЭ;

Институт теоретической и экспериментальной физики

им. А. И. Алиханова

E-mail: natanzons@mail.ru

\section{А. М. Пратусевич (А. M. Pratusevich)}

University of Liverpool, Liverpool, UK

E-mail: annap@liv.ac.uk
Представлено С. К. Ландо Принято редколлегией 22.09 .2015 